\title{
Superhydrophobic nature of nanostructures on an indigenous Australian eucalyptus plant and its potential application
}

This article was published in the following Dove Press journal:

Nanotechnology, Science and Applications

19 October 2011

Number of times this article has been viewed

\section{Gérrard Eddy Jai Poinern \\ Xuan Thi Le \\ Derek Fawcett}

Murdoch Applied Nanotechnology Research Group, Department of Physics, Energy Studies and Nanotechnology, School of Engineering and Energy, Murdoch University, Murdoch, Western Australia
Correspondence: Gérrard Poinern Murdoch Applied Nanotechnology Research Group, Department of Physics, Energy Studies and Nanotechnology, School of Engineering and Energy, Murdoch University, Murdoch,

Western Australia 6150

$\mathrm{Tel}+6 \mid 893602892$

Fax +61893606183

Email g.poinern@murdoch.edu.au

\begin{abstract}
In this preliminary study, the morphology and nanostructured features formed by the epicuticular waxes of the mottlecah (Eucalyptus macrocarpa) leaf were investigated and quantified. The surface features formed by the waxes give the leaf remarkable wetting and selfcleaning properties that enhance the plant's survival in an arid climate. This paper also provides experimental evidence of the self-assembly properties of the epicuticular waxes. Analysis of the water contact angle measurements gave a mean static contact angle of $162.00 \pm 6.10$ degrees, which clearly indicated that the mottlecah's leaf surface was superhydrophobic. Detailed field emission scanning electron microscopy examination revealed that the surface was covered by bumps approximately $20 \mu \mathrm{m}$ in diameter and regularly spaced at a distance of around $26 \mu \mathrm{m}$. The bumps are capped by nanotubules/pillars with an average diameter of $280 \mathrm{~nm}$ at the tips. Self-cleaning experiments indicated that the mottlecah's leaf could be effectively cleaned by a fine spray of water droplets that rolled over the surface picking up contaminants. Field emission scanning electron microscopy investigation of extracted epicuticular waxes revealed that the waxes were capable of self-reassembly and formed features similar to those of the original leaf surface. Furthermore, also reported is a simple technique for surface treating one side of a planar surface to produce a superhydrophobic surface that can be used as a planar floatation platform for microdevices.
\end{abstract}

Keywords: superhydrophobic, nanopillars, epicuticular waxes, self-cleaning, floatation, micro-fluidic

\section{Introduction}

The wetting of a solid material by water is a very important concept and has wide practical applications in both nature and industry. Since the discovery of the lotus (Nelumba nucifera) effect by Barthlott there has been a continued search for other similar surfaces in nature. ${ }^{1}$ Indeed, taro (Colocasia esculenta) leaves, Indian watercress (Trapaeolum majus), and even the petals of several flowers have been found to display surface structures/features at both the micrometer and nanometer scale that have similar characteristics to lotus leaves. Here, the discovery of nanopillar bunches on the leaves of an Australian indigenous eucalyptus plant called the mottlecah (Eucalyptus macrocarpa) is reported. This arid plant's cuticular membrane displays a remarkable ability to repel water and exhibits the same self-cleaning properties as the lotus leaf for materials such as carbon-black toner and silica-based Ballotini microspheres. Furthermore, the epicuticular waxes formed by this leaf have the ability to self-assemble and create both nano- and microstructures over the leaf surface.

Nature has repeatedly demonstrated a remarkable ability to adapt to changing circumstances using a wide array of forms and designs that provide unique survival 
properties for the progression of species, whether they are plant or animal in origin. With about 800 species, eucalypts constitute the most prominent genus populating the arid Australian landscape. ${ }^{2}$ With a low annual rainfall over most of the continent, these plants have adapted very well and have provided a source of food and shelter for many other indigenous species. For example, the Australian koala feeds exclusively on one particular species of eucalyptus leaves. It is only since the arrival of European settlers in the 1830s that the landscape has fundamentally changed, when the large-scale clearing of the landscape for agriculture and communities began. This has created several regions with endangered species and some eucalypts have already been placed on the endangered species list. ${ }^{3}$

The south-west corner of Australia, a biodiversity hotspot, ${ }^{4,5}$ is also the native province of the E. macrocarpa, also known as the Rose of the West or the mottlecah (Figure 1A). This plant is generally recognized by its silvery foliage and red flowers (Figure 1B and C). Because of its attractive floral arrangement, this particular eucalyptus is not an endangered species. The mottlecah's leaves have a distinctive ovate shape that can grow up to $12 \mathrm{~cm}$ long and have a silvery-grey appearance, which hint at the possibility of interesting surface structures.

In this study, the outermost cuticle layer of the mottlecah leaf was investigated for its morphology and structural features. The cuticle consists of a cutin matrix, which is composed of an insoluble, nonreactive polyester of crosslinked hydroxyl-fatty acids and hydroxyepoxy-fatty acids. In addition, there are waxes embedded in (intracuticular) and deposited on (epicuticular) the surface of the matrix. ${ }^{6}$ The composition and structure of the cuticle has been extensively reviewed in the literature by a number of authors and the reader is referred to these for further details. ${ }^{7-12}$ The surface of this type of leaf is inhomogeneous and the presence of these

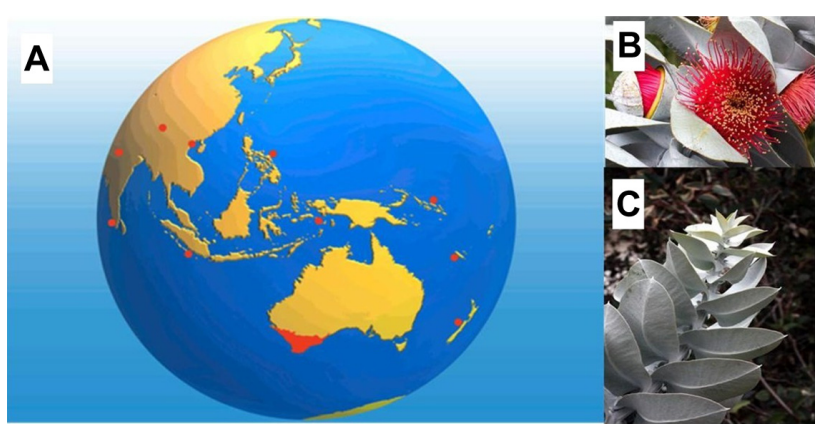

Figure I (A) Australasian biodiversity hotspots (marked in red). (B) Eucalyptus macrocarpa (mottlecah), a shrub native to the South Western Australian biodiversity hotspot. (C) Silvery leaves of the mottlecah. waxes can lead to fruits and leaves having a characteristic waxy appearance..$^{13}$ The cuticle waxes are a complex mixture of straight-chain aliphatic hydrocarbons and numerous other compounds such as secondary alcohols and $\beta$-diketones. The function of the waxes is to reduce water loss during transpiration and permit the exchange of gases needed for photosynthetic processes that support life functions. In addition, it is the epicuticular wax composition, physical structure, and crystallinity that influence the surface wettability of the leaf. There have been many extensive studies of similar epicuticular waxes' chemical composition, ${ }^{14-17}$ morphology, ${ }^{18,19}$ crystallinity, ${ }^{20-22}$ and self-assembly ${ }^{23,24}$ However, in this study, the authors concentrated on using the mottlecah's epicuticular waxes to produce a new surface treatment method and to investigate some of the plant's properties for potential application in planar floatation platforms.

Studies have shown that in the case of $N$. nucifera (sacred lotus) leaves, there is interplay between the micron-sized surface structures and the nano-sized wax crystal formations. This interplay effectively increases the surface roughness and makes the leaf surface more water repellant or hydrophobic. ${ }^{1,25}$ When water comes into contact with the leaf surface the water drops do not spread but instead form beads. The beads easily roll off the leaf and in the process remove foreign particles from its surface, thus giving the lotus leaf the added property of being self-cleaning. The wetting and self-cleaning properties of the mottlecah have been briefly mentioned. ${ }^{26}$ In this article, the size of the nanopillars, morphology, and hierarchal structure exhibited by the surface of the mottlecah leaf are quantitatively reported. In addition, the self-cleaning properties of the leaf surface with respect to carbon black and Ballotini microspheres (soda-lime glass) ${ }^{27}$ contaminants were also investigated, evaluated, and discussed. These materials were examined because they exemplify two extreme water responsive properties: in the first case, carbon black is highly hydrophobic and, in the second, Ballotini microspheres are hydrophilic. Furthermore, a simple and efficient replication technique that involves removing the epicuticular waxes from the surface of the mottlecah's leaf and then depositing them onto a planar surface (microscope glass slide) is also reported. The waxes then reassemble on the planar surface to mimic the complex three-dimensional geometry of the nano/microstructures originally seen on the leaf. In addition, the new surface structure also inherits the superhydrophobic properties of the leaf surface. Similar studies have reported making artificial hydrophobic surfaces using a variety of materials such as carbon nanotubes, ${ }^{28,29}$ glasses, ${ }^{30}$ metals,,$^{31,32}$ polymers ${ }^{33,34}$ and waxes. ${ }^{22,35}$ This work 
is the first to use the self-reassembled mottlecah epicuticular waxes on a surface to fabricate a planar floatation platform capable of supporting a microdevice. The simplicity of the technique makes it possible to cover a reasonably large area, in this case a standard laboratory glass slide, using materials and equipment found in a general laboratory at ambient conditions without the need for complex equipment.

When a water drop wets a normal smooth surface it forms a bead with a low internal contact angle, $\theta<90^{\circ}$. Measurement of the contact angle provides an inverse measure of the surface wettability: the smaller the contact angle, the greater the wettability. A nonwetting or hydrophobic drop forms a bead with a high internal contact angle that is between $90^{\circ}$ and $150^{\circ}$. When the internal contact angle exceeds $150^{\circ}$ the surface is known as a "superhydrophobic" surface (see Figure 2). The surface of Nelumba nucifera can produce a contact angle of $162^{\circ}$, which makes it a superhydrophobic surface. Following the pioneering work of Young, the ideal contact angle $\theta$ for a normal smooth surface was defined as ${ }^{36}$

$$
\cos \theta=\frac{\gamma_{s v}-\gamma_{s l}}{\gamma_{l v}}
$$

where $\gamma_{s v}$ denotes the surface tension of the solid-vapor interface, $\gamma_{s l}$ denotes the surface tension of the solid-liquid interface and $\gamma_{v v}$ denotes the surface tension of the liquidvapor interface. In this equation, the surface is assumed to be ideal, which means that the surface is perfectly smooth, chemically homogeneous, rigid, and flat. In the ideal case, the advancing and receding contact angles will remain the same. However, on real surfaces the advancing and receding contact angles may differ, resulting in contact angle hysteresis, with surface roughness enhancing this effect. ${ }^{37}$ The effect of surface roughness on the contact angle was further investigated by Wenzel $^{38}$ and later by Cassie and Baxter. ${ }^{39}$ The Wenzel model describes the homogeneous wetting

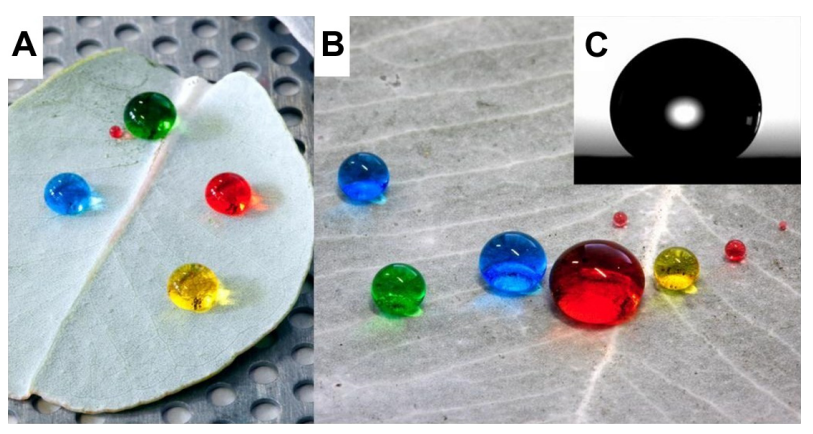

Figure 2 (A) Dye-colored water drops, with high contact angle on a mottlecah leaf. (B) A size selection of dye-colored water drops possible on a mottlecah leaf. (C) Picture of a sessile water drop used during contact angle measurements. regime, which assumes a rough material will have a high surface area resulting from the numerous surface furrows, and liquid from the water drop will fill up the furrows. The ratio of the solid-liquid area $\mathrm{A}_{s l}$ over the projected area $\mathrm{A}_{p}$ gives the roughness factor $R_{f}=\mathrm{A}_{s l} / \mathrm{A}_{p}$. When the roughness factor is multiplied by Young's ideal contact angle, the result produces the apparent contact angle for a real surface:

$$
\operatorname{Cos} \theta_{w}=R_{f} \cos \theta
$$

The Cassie and Baxter model is more complex and predicts the apparent contact angle of a heterogeneous surface. In this model two phases are involved. This is due to the liquid not completely filling the furrows and, as a result, air is trapped in the rough surface..$^{40-42}$ The two interface areas are the liquid-solid interface, $F_{l s}$ and liquid-vapor interface, $F_{l v}$. The contact angle for a heterogeneous surface:

$$
\cos \theta_{c b}=R_{f} F_{l s} \cos \theta-F_{l v}
$$

\section{Materials and methods Contact angle measurements}

A wide selection of healthy mottlecah leaves was harvested, ranging from young to mature leaves and from various locations on the plant. Generally, five locations were selected (top, north, south, east, and west) and, on average, eleven leaves were taken from each of the locations. Both the adaxial and abaxial sides of the leaf were examined, the adaxial surface having the greater surface concentration of waxes. Initial visual inspection of water droplets sprayed on the abaxial surface of the leaf indicated less beading and a lower degree of spherical morphology during water-droplet formation compared with those on the adaxial surface, which produce a high degree of beading and spherical water droplets. Since one of the objectives of this research was to test whether this wax was capable of reassembly and use in a planar floatation platform, the adaxial side of the leaf became the focus of the study since it provided the greatest source of readily available surface waxes.

Depending on leaf size, a selection of square samples from various locations over the adaxial surface of the leaf was chosen to give a representative surface structure of the whole leaf. On average, six sample locations were studied, with the tip, middle, and base of the leaf being examined. Each of the square leaf samples was cut and set onto a microscope slide using doublesided tape to produce a flat and even surface. Suitably dyed droplets of $10-15 \mu \mathrm{L}$ Milli-Q ${ }^{\circledR}$ water (Barnstead Ultrapure Water System D11931; Thermo Scientific, Dubuque, IA) 
(18.3 $\left.\mathrm{M} \Omega \mathrm{cm}^{-1}\right)$ were deposited from a syringe in four different positions on each specimen to ensure that the drops were not sitting on a contaminated or damaged part of the leaf. The static measurement of the contact angle (CA) was performed using a CAM 100 system from KSV Instruments Inc (Biolin Scientific, Helsinki, Finland). Snapshots of each drop were taken at one per second over 4 seconds to reduce the influence of oscillations. The snapshot that produced the most comparable angle on both the left- and right-hand sides for each drop was used to calculate the CA (Figure 2C). Measurements were taken of each of the four droplets on each square sample. On average, 24 droplets were examined for each leaf. From these measurements the mean and standard deviation for the static CA were subsequently calculated and found to be $162.00^{\circ} \pm 6.10^{\circ}$.

\section{Optical microscopy}

Before optical microscopy was carried out, samples of the leaf structure were prepared, treated, and then mounted on glass slides. The sample preparation procedure started with small samples of leaf tissue being cut from the mottlecah leaf. The samples were then placed into sample vials containing a solution of glutaraldehyde and allowed to soak overnight at $4^{\circ} \mathrm{C}$. The following day the glutaraldehyde solution was removed and Dalton's chrome osmic acid was added. The second solution was allowed to stand for 1 to 1.5 hours at $4^{\circ} \mathrm{C}$. At regular intervals during this time, the solution was agitated for 20 seconds. At the end of the standing period the leaf samples were washed in several fresh solutions of $70 \%$ alcohol before being placed in a 60:40 solution of propylene oxide/epoxy resin for 1 hour. (All analytical grade chemicals were purchased from Sigma-Aldrich [Castle Hill, NSW, Australia] and used without further purification.) The samples were then placed on a rotator (Murdoch University Rotation Platform; Murdoch University, Perth, Australia) overnight. The next day the leaf samples were embedded into capsules, using fresh epoxy resin, and then placed into an electric oven (Napco 5831 Vacuum Oven; Precision Scientific, Inc, Chicago, IL) for thermal treatment and baked at $60^{\circ} \mathrm{C}$ for 24 hours to suitably harden the samples. For optical microscopy the treated leaf sections were too thick, so they were cut into thin sections, approximately $1 \mu \mathrm{m}$ thick, stained with toluidine blue, and then allowed to dry. The leaf sections were then mounted on glass slides for examination.

\section{Field emission scanning electron microscopy (FESEM) measurements}

A Zeiss 1555 VP-FESEM (Carl Zeiss MicroImaging GmbH, Gottingen, Germany) electron microscope was used to investigate the detailed surface morphology of both the top and bottom of the mottlecah leaf samples. Another representative group of leaves were selected and dried in a vacuum oven (Napco 5831 Vacuum Oven; Precision Scientific, Inc) to reduce their water content prior to coating the leaf surfaces with a fine gold layer. The FESEM was operated at $6.0 \mathrm{mPa}$ with a $15 \mathrm{kV}$ accelerating voltage for images without Ballotini microspheres and a voltage of $5 \mathrm{kV}$ for images with Ballotini microspheres.

The software used for the dimensional analysis of images was GIMP (v 2.6; The GIMP Development Team). A representative image was used to take a sample of 50 random measurements using the measuring tool in the program. The number of pixels across the scale bar for the image was used to determine the distance represented by 1 pixel. By measuring the number of pixels across each leaf surface feature, such as a tubule or a bump, the total representative distance could be determined. The data were then used to calculate the mean and standard deviation for each feature located on the surface of the leaf sample. In addition, FESEM microscopy was used to examine the adaxial surface of the mottlecah leaf before and after the wax extraction procedure. This technique ensured that only the epicuticular waxes were collected and the action of the chloroform had not damaged the underlining cellular structure.

\section{Self-cleaning experiment using Ballotini microspheres}

The leaf surface covering was prepared by first coloring the Ballotini microspheres (Potters Europe, Barnsley, UK) using a standard food dye. Ballotini microspheres are composed of soda-lime glass ${ }^{27}$ and are extremely hydrophilic, which makes them suitable for color dye adsorption. The microspheres were first soaked in a diluted dye solution for 2 hours, before being vacuum dried to remove all moisture. The microspheres were then ground with a mortar and pestle to remove any microsphere clumps created by the dye process. The resulting powder was then finely distributed over the entire surface of a clean, fresh mottlecah leaf, with any excess powder being shaken off. The leaf was then oriented with a slight dipping angle relative to the horizontal plane and a droplet of Milli-Q water was placed onto the top left hand portion of the leaf surface using a glass pipette fitted with a rubber bulb. Under the influence of gravity, this single droplet rolled across the surface of the leaf producing a clean pathway through the distributed microspheres. The motion of the droplet was filmed using a small standard digital camera (Olympus FE220; Olympus Corp, Tokyo, Japan). The sequential frames were then extracted to show the cleaning action of the droplet. After the self-cleaning experiment, a scalpel was used to excise a small section of the pathway from the leaf. This section was then mounted on a scanning electron microscopy (SEM) stub using 
conductive carbon tape and then the leaf section was sputter coated with a fine gold layer for FESEM imaging. GIMP software was then used to produce false-color FESEM images to highlight the pathway through the Ballotini microspheres.

\section{Self-cleaning experiment using carbon-black toner}

The self-cleaning action of the mottlecah leaf was also investigated using standard carbon-black toner obtained from a standard laser printer cartridge. This carbon material was selected due to its highly hydrophobic nature and its ability to be picked up by rolling water droplets and form water marbles. A clean mottlecah leaf was selected, harvested and coated in carbon-black toner. The leaf was oriented with a slight dipping angle relative to the horizontal plane so that the water drops could easily roll off the leaf. A number of short fine sprays of water (from a normal handheld and finger-pump garden spray mister) were sprayed over the surface of the leaf. One complete trigger movement gave one full squirt, which produced $1.10 \mathrm{~mL}$ of water as a fine mist. The motion of the water spray over the surface of the leaf and the interaction between the water droplets and the carbon-black toner was filmed using a small standard digital camera. The sequential frames were then extracted to show the self-cleaning effect of the leaf.

\section{Self-assembly of epicuticular waxes on glass slides}

A precleaned mottlecah leaf was selected and a solution of chloroform used to wash the adaxial leaf surface to remove the surface waxes. The washing procedure consisted of slowly pouring a $50 \mathrm{~mL}$ solution of chloroform over the entire inclined leaf, collecting the run off in a small beaker. During the 30-second washing period, the chloroform efficiently extracted the epicuticular waxes from the leaf surface. Subsequent SEM analysis revealed that the waxes had indeed been removed from the leaf surface leaving the underlying cutin undamaged. A droplet of the collected wax/chloroform solution was then deposited onto a clean glass slide using a small pipette and the slide was allowed to stand until the chloroform had completely evaporated, leaving the wax crystals behind. Several slides were prepared using this simple procedure, thus providing a selection of samples for FESEM examination; typical images can be seen in Figure 6A-D.

\section{Surface treatment of planar surface and water interaction experiments}

The solution of wax/chloroform collected from the washing procedure described above was then used for treating the surface of several glass slides. In each case, one of the large planar surfaces of the glass slide was covered liberally with a layer of the wax/chloroform solution using a small pipette. The coated glass slides were then allowed to air dry in ambient atmospheric conditions within the laboratory. During this time, the chloroform evaporated, allowing a reassembling epicuticular wax layer to form. After 2 hours, each glass slide had become opaque and had a frost-like appearance that indicated the presence of solid wax structures over the surface.

The first experiment involved inserting a glass slide vertically into a beaker of water to see the interaction of the slide with the water meniscus. First, an untreated glass slide (control) was inserted into the water solution and the meniscus photographed. Second, a glass slide surface treated with wax on one side was inserted vertically into the water solution. In both cases, the slide was positioned with the edge of the slide facing toward the camera, the water meniscus on both sides of the slide was photographed (Figure 7A and B) using a Canon EDS 600D (Canon Inc, Tokyo, Japan) digital camera (Canon macro lens, EF100 mm, 1:28 USM; image specifications: shutter 1/60, aperture 4.0, ISO 400).

In the second experiment, the effect of the surface treatment in improving the floatation capacity of a planar surface was investigated. In this experiment, two similar microscope glass slides were used; the first had no surface treatment as a control, while the second received a surface layer of wax on one side. Both glass slides were then placed onto a large sheet of $125 \mathrm{~mm}$ diameter Whatman cellulose filter paper (Whatman plc, Kent, UK). The surface-treated glass slide had its waxed side placed face down on the filter paper, then the filter paper and slides were carefully placed onto a water surface contained in a large beaker. The cellulose filter paper then slowly became waterlogged and started sinking, leaving the slides to float evenly on the water surface. Masses were lightly placed on each glass slide until the untreated glass slide sank. After placing one mass (1.00 g) on both glass slides the untreated glass slide sank, leaving the wax-treated slide floating. A further mass was then added to the treated glass slide, giving it a total mass of $2.00 \mathrm{~g}$ (Figure 7C). This procedure was continued until a maximum load of $4.00 \mathrm{~g}$ was achieved.

\section{Results and discussion}

The mottlecah has a remarkable leaf surface structure that gives it superhydrophobic properties. This property can easily be seen when dye-colored water drops are placed on the leaf surface (Figure 2A and B). The composition, physical 
structure, and crystallinity of the epicuticular waxes produces $20 \mu \mathrm{m}$ tall bumps capped with bunches of nanotubules/pillars over the outer surface of the cuticle. The presence of these bumps was first seen under optical microscopy examination of the leaf cross-section (see Figure 3A). Subsequent FESEM analysis revealed:

- The mottlecah leaves are covered with a fine array of small bumps, capped with nanotubules/pillars. The bumps have regular and periodic structures which are typically seen over the entire adaxial surface, (Figure 3C).

- The bumps are on average $20 \mu \mathrm{m}$ in diameter and are spaced at around $26 \mu \mathrm{m}$ between centers.

- The nanotubules/pillars have an average diameter of $280 \mathrm{~nm}$ at their tips (Figure 3D). These unique surface features produce a surface of low water wettability, resulting in the formation of spherical water droplets.

To investigate the interaction between the water drop and the leaf's surface, water-droplet contact measurements were taken on a wide range of leaf samples. A typical image of a sessile water droplet used to make contact angle measurements is presented in Figure 2C. Analysis of the samples revealed that the mean and standard deviation for the static contact angle was calculated to be $162.00 \pm 6.10^{\circ}$, which makes the mottlecah leaf superhydrophobic. This high contact angle permits the spherical water droplets to roll off inclined leaf surfaces and pick up surface contaminants in the process.

The self-cleaning properties of the mottlecah leaf surface were investigated using Ballotini microspheres and

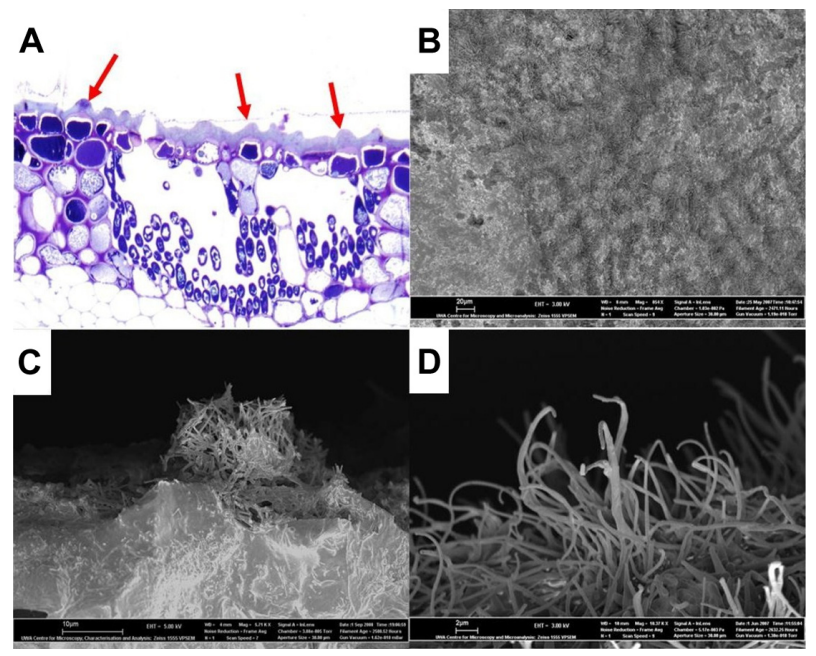

Figure 3 (A) Optical microscopy of a stained mottlecah leaf with surface bumps. (B) Electron microscopy micrograph of mottlecah leaf surface. (C) High magnification scan image of micron sized bumps capped with nanotubules/pillars. (D) Fine nanotubules/pillars on leaf surface.

Notes: Scale bars: B, $20 \mu \mathrm{m}$; C, $10 \mu \mathrm{m}$; D, $2 \mu \mathrm{m}$. carbon-black toner material. In the first case, the self-cleaning properties were examined using a single rolling water drop on a leaf surface totally covered with Ballotini microspheres, which are extremely hydrophilic. Figure $4 \mathrm{~A}-\mathrm{C}$ demonstrate that the rolling water drop is clearing a pathway through the Ballotini microspheres. This was confirmed by the colorenhanced FESEM images presented in Figure 4D-F, which reveal that there was a significant reduction in microsphere concentration where the water drop had rolled and a pathway had been made. The second material used to investigate the self-cleaning ability of the mottlecah leaf was carbon-black toner, which was deposited on the leaf's surface so that the leaf appeared totally black (Figure 5A). Then a series of short fine water sprays were aimed at the surface of the leaf. After receiving one full squirt and half of a second squirt ( $\sim 1.65 \mathrm{~mL}$ in total), the water droplets started to pick up the carbon particles (Figure 5B) and after 2.5 squirts $(\sim 2.75 \mathrm{~mL}$ in total) the formation of black water marbles resulting from the rolling water drops and carbon particles was seen (Figure 5C). After four squirts ( $\sim 40 \mathrm{~mL}$ in total) the leaf surface was completely cleaned (Figure 5D). The superhydrophobic nature of the leaf structure has allowed the hydrophobic surface contaminants to be efficiently washed off. The carbon material was used because it is a hydrophobic material and effectively demonstrated that particles such as dust can be picked up and moved by water droplets. This behavior is clearly visible in Figure 5C with the formation of water marbles.

It is interesting to note that the majority of superhydrophobic plant structures reported to date are mostly found in or close to an aquatic setting. This environmental factor has been suggested to be the main driver behind the superhydrophobic leaf surface adaptation. The mottlecah is not in this category. It is not yet clear why this Australian plant developed these leaf surface features, but it may well be the result of another facet of an evolutionary adaptation. As the Australian climate changed from tropical/subtropical to arid, the plant's survival depended on sourcing and effectively managing its water usage. The surface features, composed of epicuticular waxes and the cuticle matrix, limit the loss of water and substances from the leaf's internal tissues. The superhydrophobic nature of the leaf surface structure causes spherical water droplets to form and roll off the leaf toward the ground at the base of the plant. This channeling effect is similar to the way water is channeled by the Stenocara beetle's back. ${ }^{43}$ In addition, the mottlecah's nanotubular pillars may also assist the cuticle in reducing ultraviolet radiation damage in a similar way to some xerophytes. ${ }^{44}$ 


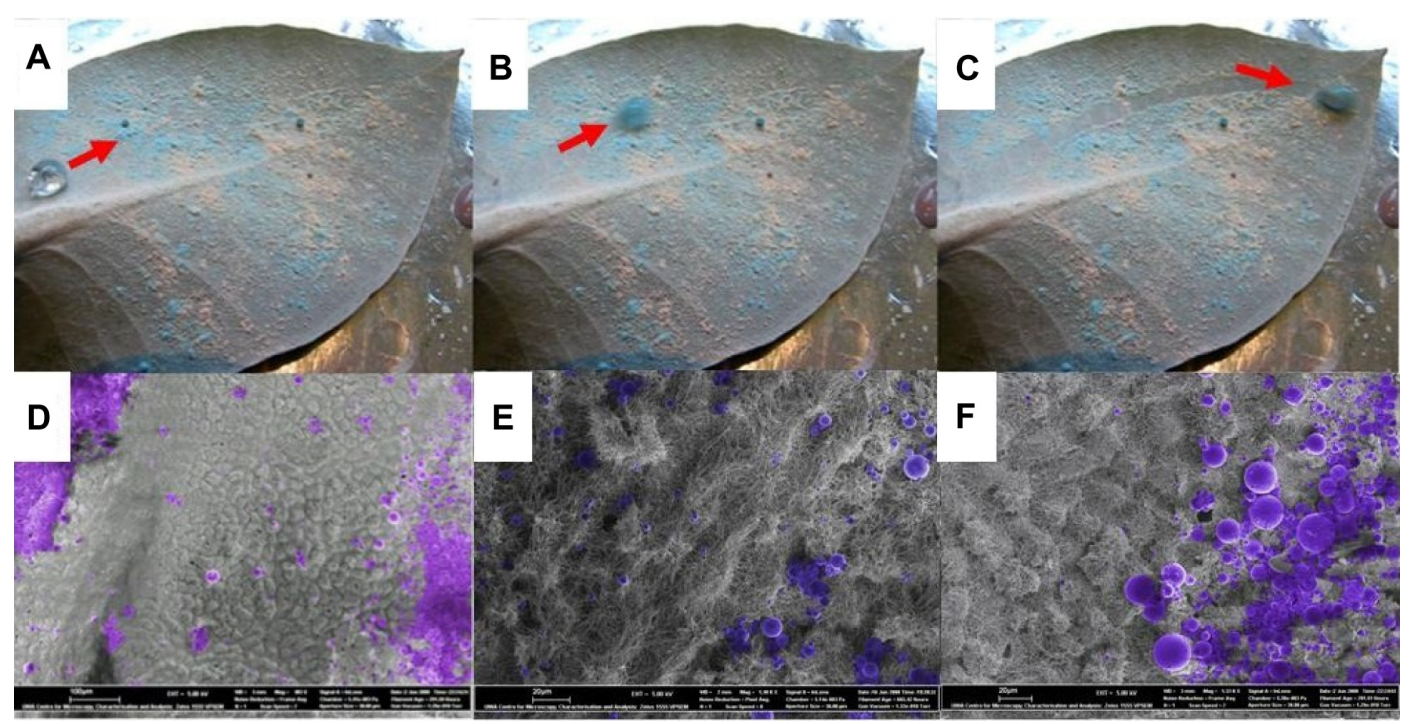

Figure 4 (A-C) Water drop rolls across Ballotini microsphere-coated mottlecah leaf and clears pathway. (D-F) FESEM images of the pathway produced by the self-cleaning action of the water drop and the leaf.

Notes: Scale bars: D, $100 \mu \mathrm{m}$; E, $20 \mu \mathrm{m} ; \mathbf{F}, 20 \mu \mathrm{m}$.

Abbreviation: FESEM, field emission scanning electron microscopy.

An interesting property of the epicuticular waxes produced by the mottlecah leaf, observed in this study, is their ability to self-reassemble. When these waxes were dissolved and exacted from the leaf surface using chloroform, a droplet of the resultant mixture was deposited onto a clean glass slide. Several slides were prepared in this manner and then allowed to stand for the chloroform to evaporate, leaving the wax crystals behind. ${ }^{22}$ The coated samples were then scanned using FESEM; the subsequent images revealed that the waxes had reassembled themselves into bumps and nanotubules/ pillars that were originally seen on the leaf's surface. This can be seen in Figure 6B, which clearly shows the bump formation on the glass slide and the higher magnification

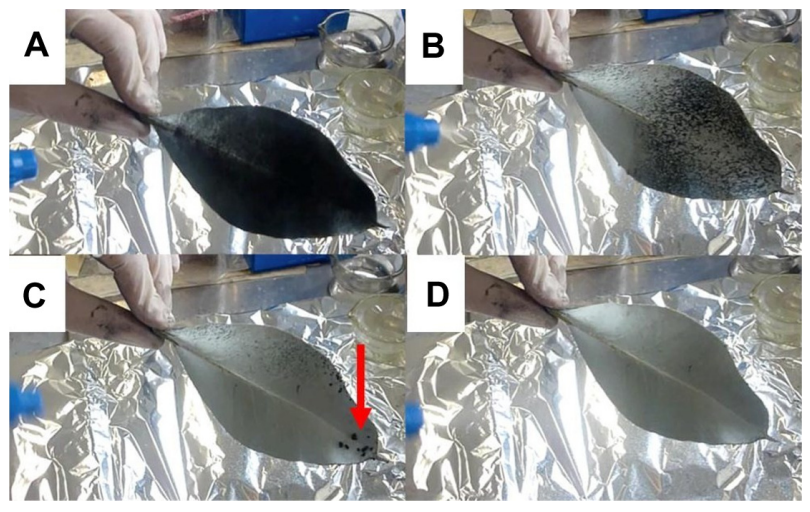

Figure 5 (A) Mottlecah leaf coated with carbon-black toner material. (B) Water being sprayed (one full squirt and first half of second squirt, $\sim 1.65 \mathrm{~mL}$ ). (C) End of third squirt $(3.30 \mathrm{~mL})$ produces rolling black marbles of water (arrow). (D) At the end of fourth squirt $(4.40 \mathrm{~mL})$, the carbon-black material is completely removed from the leaf surface. images presented in Figure 6C and D show the formation of nanotubules/pillars on the bumps. Comparing these images with those of the leaf surface in Figure 3C and D, a similar geometry and structure can be seen.

Having confirmed the replication of the epicuticular wax structures on a glass slide, the next stage in the study was to investigate the wax's potential application in a planar floatation platform for a variety of possible microfluidic devices. The surface treatment technique used was simple, flexible, and effective. It also had the advantage of using materials and equipment found in a general laboratory at ambient conditions without the need for complex equipment.

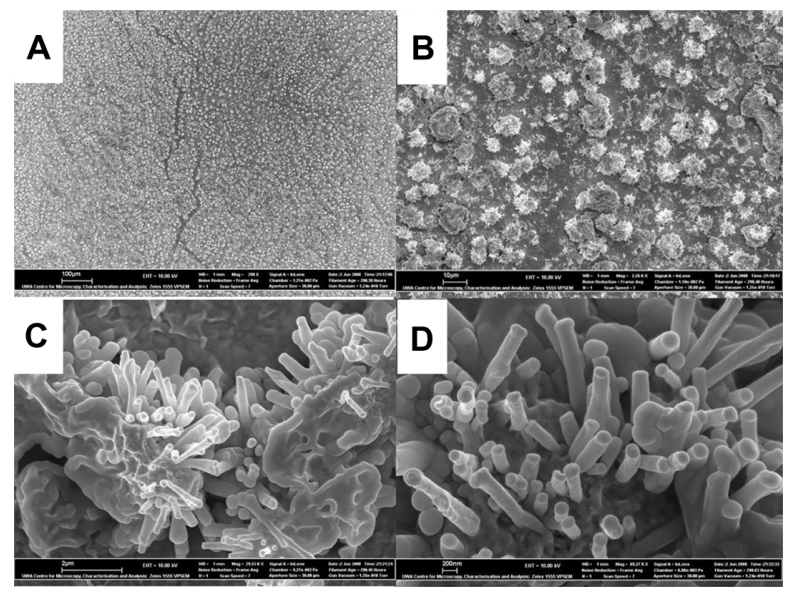

Figure 6 Field emission scanning electron microscopy images showing: (A) regularly spaced $20 \mu \mathrm{m}$ diameter bump structures on leaf surface; (B) enlarged image of bump structures; (C) nanotubule/pillar formations on micron sized bumps; and (D) enlarged image of nanotubules/pillars on bumps.

Notes: Scale bars: A, $100 \mu \mathrm{m}$; B, $10 \mu \mathrm{m}$; C, $2 \mu \mathrm{m}$; D, $200 \mathrm{~nm}$. 
The simplicity of the technique also made it possible to cover a reasonably large area, in this case a standard laboratory glass slide, without the need for specialized coating techniques.

One prominent result of the surface treatment technique was the change in appearance of the water meniscus when glass slides were inserted vertically into a solution of water. When an untreated glass slide was inserted into a water solution the meniscus moves up the glass surface indicating that the glass surface is water wettable (Figure 7A). When a similar glass slide surface, treated on one side (left-hand side in Figure 7B), was immersed in the water solution, a different meniscus effect was seen. The image effectively demonstrates the interaction between the water and the treated glass slide, where the left-hand side (wax surface treatment), has a downward-bending meniscus indicating the nonwetting property of the wax-treated surface. On the other side of the glass slide (untreated surface) the water meniscus bends upward as in the case of the control. Furthermore, when the glass slides are placed horizontally onto the water surface, the added buoyancy support of the wax on the treated slide meant that it was able to carry a greater load than the control slide. The control slide was only capable of minimal support and the addition of a $1 \mathrm{~g}$ mass resulted in the slide sinking. The maximum load carried by the treated slide was $4 \mathrm{~g}$, thus

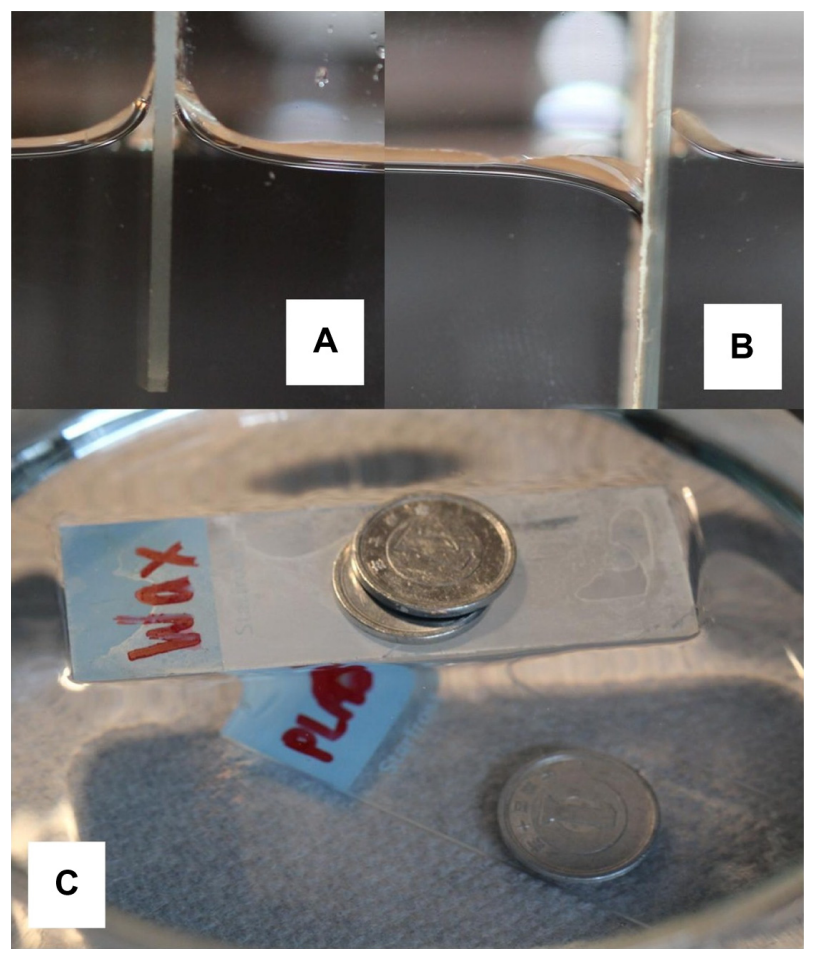

Figure 7 (A) Untreated microscope glass slide immersed in water. (B) Glass slide with left-hand side coated with mottlecah leaf wax. (C) Floating glass slide with waxed surface in contact with water above a sunken untreated glass slide. demonstrating the positive buoyancy support offered by this surface treatment (Figure 7C).

\section{Conclusion}

This preliminary study shows that the surface features and properties of the mottlecah's leaf epicuticular waxes can be effectively replicated. The reassembled wax can be used to provide buoyancy support for a planar platform as well as a potential application for coating channels in microfluidic devices. If the surface properties of the mottlecah leaf could be efficiently reproduced in a similar manner to those of the sacred lotus leaf (which has been artificially reproduced to manufacture self-cleaning coatings for buildings), then it should be possible to reproduce the surface properties of the mottlecah leaf in similar applications. The unique surface properties of mottlecah leaf wax have the potential to be applied to problems such as fluid drag reduction, enhancing water supporting forces, and antibiofouling of ships' hulls to name just few. ${ }^{45}$

\section{Acknowledgments}

The authors would like to thank Dr B Richmond for use of the KAV CA measurement XP1 system for the contact angle measurements and MrT Thomson, research assistant, in performing the surface cleaning experiments. Dr D Fawcett wishes to thank the Bill and Melinda Gates Foundation for a research fellowship at the Murdoch Applied Nanotechnology Research Group. The authors would also like to thank Mr Peter Fallon from the Electron Microscopy Unit at Murdoch University, Perth, for his assistance with the optical microscopy of the leaves.

\section{Disclosure}

The authors report no conflicts of interest in this work.

\section{References}

1. Barthlott W, Neinhuis C. Purity of the sacred lotus, or escape from contamination in biological surfaces. Planta. 1997;202(1):1-8.

2. Brooker MIH, Kleinig DA. Field Guide to Eucalypts: South-Western and Southern Australia. 1st ed. Sydney: Bloomings Books; 2001.

3. Pryor LD, Briggs JD. Australian Endangered Species: Eucalypts. 1st ed. Canberra: Commonwealth of Australia; 1981.

4. Cincotta RP, Wisnewski J, Engelman R. Human population in the biodiversity hotspots. Nature. 2000;404(6781):990-992.

5. Conservation International. Biodiversityhotspots.org [homepage on the Internet]. Updated October 23, 2008. http://www.biodiversityhotspots. org/. Arlington, VA: Conservation International; 2007. Accessed March 30, 2011.

6. Dominguez E, Heredia Guerrero JA, Heredia A. The biophysical design of plant cuticles: an overview. New Phytol. 2011;189(4):938-949.

7. Riederer M, Schonherr J. Development of plant cuticles: fine structure and cutin composition of Clivia miniata Reg. Leaves. Planta. 1988; 174:127-138.

8. Rieder M, Muller C. Biology of the Plant Cuticle. Oxford: Blackwell; 2006. 
9. Jeffree CE. Structure and ontogeny of plant cuticles. In: Kerstiens G. editor. Plant Cuticle: An Integrated Functional Approach. Oxford, UK: BIOS Scientific Publishers; 1996:33-82.

10. Walton TJ. Waxes, cutin and suberin. Methods Plant Biochem. 1990; 4:105-158.

11. Bargel H, Koch K, Cerman Z, Neinhuis C. Structure-function relationships of the plant cuticle and cuticular waxes-a smart material? Funct Plant Biol. 2006;33:893-910.

12. Heredia A. Biophysical and biochemical characteristics of cutin, a plant barrier biopolymer. Biochim Biophys Acta. 2003;1620:1-7.

13. Storey R, Price WE. Microstructures of the skin of d'Agen plums. Sci Hortic (Amsterdam). 1999;81(3):279-286.

14. Baker EA. Chemistry and morphology of plant epicuticular waxes. In: Cutler DF, Alvin KL, Price CE, editors. The Plant Cuticle. London: Academic Press; 1982:139-166.

15. Holloway PJ, Jeffree CE, Baker EA. Structural determination of secondary alcohols from plant epicuticular waxes. Phytochemistry. $1976 ; 15: 1768-1770$

16. Buschhaus C, Herz H, Jetter R. Chemical composition of the epicuticular and intracuticular wax layers on adaxial sides of Rosa canina leaves Ann Bot (London). 2007;100:1557-1564.

17. Wen M, Buschhaus C, Jetter R. Nanotubules on plant surfaces: chemical composition of epicuticular wax crystals on needles of Taxus baccata $\mathrm{L}$. Phytochemistry. 2006;67(16):1808-1817.

18. Noga G, Knoche M, Wolter M, Barthlott W. Changes in leaf micromorphology induced by surfactant application. Angew Bot. 1987;61:521-528.

19. Gomez-Campo C, Tortosa ME, Tewari I, Tewari JP. Epicuticular wax columns in cultivated Brassica species and in their close wild relatives. Ann Bot (London). 1999;83(5):515-519.

20. Ensikat HJ, Boese M, Mader W, Barthlott W, Koch K. Crystallinity of plant epicuticular waxes: electron and X-ray diffraction studies. Chem Phys Lipids. 2006;144:45-59.

21. Meusel I, Barthlott W, Kutzke H, Barbier B. Crystallographic studies of plant waxes. Powder Diffr. 2000;15:123-129.

22. Dora SK, Wandelt K. Recrystallization of tubules from natural lotus (Nelumbo nucifera) wax on a $\mathrm{Au}(111)$ surface. Beilstein J Nanotechnol. 2011;2:261-267.

23. Zhang S. Fabrication of novel biomaterials through molecular selfassembly. Nat Biotechnol. 2003;21:1171-1178.

24. Neinhuis C, Koch K, Barthlott W. Movement and regeneration of epicuticular waxes through plant cuticles. Planta. 2001;213:427-434.

25. Furstner R, Barthlott W. Wetting and self-cleaning properties of artificial super hydrophobic surfaces. Langmuir. 2005;21(3):956-961.

26. Barthlott W, Neinhuis C. Characterization and distribution of waterrepellent, self-cleaning plant surfaces. Ann Bot (London). 1997;79(6): $667-677$.
27. Potters Europe, home page on the Internet. Available at: http://www. potterseurope.com [updated February 14, 2011]. Accessed March 11, 2011.

28. Lau KKS, Bico J, Teo KBK, et al. Superhydrophobic carbon nanotube forests. Nano Lett. 2003;2:1701-1705.

29. Li HJ, Wang XB, Song YL, et al. Super "amphiphobic" aligned carbon nanotube films. Angew Chem. 2001;113(9):1793-1796.

30. Ogawa K, Soga M, Takada Y, Nakayama I. Development of a transparent and ultrahydrophobic glass plate. Jpn J Appl Phys. 1993; 32:L614-L615.

31. Qian BT, Shen ZQ. Fabrication of superhydrophobic surfaces by dislocation selective chemical etching on aluminium, copper and zinc substrates. Langmuir. 2005;21(20):9007-9009.

32. Larmour IA, Bell SEJ, Saunders GC. Remarkably simple fabrication of superhydrophobic surfaces using electroless galvanic deposition. Angew Chem Int Ed. 2007;46(10):1710-1712.

33. Ming W, Wu D, van Benthem R, de With G. Superhydrophobic films from raspberry-like particles. Nano Lett. 2005;5(11):2298-2301.

34. Jung DH, Park IJ, Choi YK, Lee SB, Park HS, Ruhe J. Perfluorinated polymer monolayers on porous silica for materials with super liquid repellent properties. Langmuir. 2002;18:6133-6139.

35. Shibuichi S, Yamamoto T, Onda T, Tsujii K. Super water and oil repellent surfaces resulting from fractal structure. J Colloid Interface Sci. 1998;208:287-294.

36. Young T. An essay on the cohesion of fluids. Phil Trans R Soc London. 1805;95:65-87.

37. Patankar NA, He B, Lee J. Contact angle hysteresis on rough hydrophobic surfaces. Colloid Surface A. 2004;248(1-3):101-104.

38. Wenzel RN. Resistance of solid surfaces to wetting by water. Ind Eng Chem. 1936;28(8):988-994.

39. Cassie ABD, Baxter S. Wettability of porous surfaces. Trans Faraday Soc. 1944;40:546-551.

40. Bhushan B, Jung YC. Wetting study of patterned surfaces for super hydrophobicity. Ultramicroscopy. 2007;107(10-11):1033-1041.

41. Swain PS, Lipowsky R. Contact angles on heterogeneous surfaces: A new look at Cassie's and Wenzel's laws. Langmuir. 1998;14(23): $6772-6780$.

42. Whyman G, Bormashenko E, Stein T. The rigorous derivation of Young, Cassie-Baxter and Wenzel equations and the analysis of the contact angle hysteresis phenomenon. Chem Phys Lett. 2008;450(4-6):355-359.

43. Parker AR, Lawrence CR. Water capture by a desert beetle. Nature. 2001;414(6859):33-34.

44. Bhushan B. Biomimetics: lessons from nature - an overview. Phil Trans $R$ Soc A. 2009;367(1893):1445-1486.

45. Zhang X, Shi F, Nui J, Jiang Y, Wang Z. Super hydrophobic surfaces: from structural control to functional application. J Mater Chem. 2008; $18: 621-633$
Nanotechnology, Science and Applications

\section{Publish your work in this journal}

Nanotechnology, Science and Applications is an international, peerreviewed, open access journal that focuses on the science of nanotechnology in a wide range of industrial and academic applications. It is characterized by the rapid reporting across all sectors, including engineering, optics, bio-medicine, cosmetics, textiles, resource sustainability

\section{Dovepress}

and science. Applied research into nano-materials, particles, nanostructures and fabrication, diagnostics and analytics, drug delivery and toxicology constitute the primary direction of the journal. The manuscript management system is completely online and includes a very quick and fair peer-review system, which is all easy to use. 